NOTULES HYDRAULIQUES

HYDRAULIC BRIEFS

\title{
Débit d'une grille par en dessous
}

\section{Discharge passing through a bottom grid}

\begin{abstract}
Débit dúrivé et débit déversé par une grille de prise d'eau sur le fond d'un torrent. - Aspect thérique schématique du problème. - Tentative de résolution par approximations successives.
\end{abstract}

Les prises des torrents de montagne sont, en général, équipées au moyen d'une grille à prise par en dessous, l'eau déversée passant par-dessus et l'eau dérivée par-dessous.

On est, en général, très embarrassé quand on a à déterminer les dimensions de ces grilles, et on ne sait pas trop sur quoi se baser. Toutefois, il ne peut être question de s'en tenir aux stricts résultats d'une étude théorique quelle qu'elle soit : on devra notamment prendre une sécurité plus ou moins importante dans la surface adoptée, liée aux possibilités de bouchage partiel des grilles par des branches, des feuilles et des cailloux.

Nous allons examiner comment on pourrait avoir au moins une idée du débit dérivé.

\section{$1^{\circ}$ Considérations théorigues}

Soit $q$ le débit par $\mathrm{ml}$ de largeur qui est déversé, c'est-à-dire non capté, en un point d'abscisse $x$, complé suivant la pente.

Nous supposerons connus le tirant d'eau et la vitesse au droit de la section où commence Ia grille. Il en résultera une charge $H$ en cette scetion égale à :

$$
h+\frac{b^{2}}{2 g}=\mathrm{H}
$$

\begin{abstract}
Discharge flowing over, and discharge diverted by, an intake rack on bed of mountain torrent. -Theoretical, diagrammatic view of the problem.-An endeavour to solve it by successive approximations.
\end{abstract}

Nous supposerons que l'écoulement se fait sans perte de charge, tant pour le débit déversé que pour le débit capté. Cette hypothèse semble bien correspondre à la réalité et est, en tout cas, conforme aux hypothèses classiques des calculs hy-



drauliques analogues. Nous appliquerons séparément le théoreme des forces vives à chacun des écoulements.

a) Eau déversée. - Soit $h$ le tirant d'eau à l'abscisse $x$ :

Ia conservation de l'énergie serail :

$$
x \sin \alpha+\mathrm{H}=\frac{t^{2}}{2 g}+h \cos \alpha
$$


soit :

$$
\frac{q^{2}}{2 g h^{2}}=\mathrm{H}+x \sin \alpha-h \cos \alpha
$$

b) Eau dérivée. - $m$ étant un coefficient de contraction, le débit par in de large vaudra, d'après le théorème des forces vives :

$$
\begin{aligned}
v & =\sqrt{2 g h \cos \alpha} \\
d q & =m \sqrt{2 g h \cos \alpha} d x
\end{aligned}
$$

Ces deux équations supposent :

- La première, que la vitesse, perpendiculairement au plan de la grille, est due uniquement à la pression.

Cette condition doit cesser d'être réalisée quand le tirant d'eau atteint une valeur trop faible, bien difficile à déterminer théoriquement.

- La seconde, que le coefficient de contraction est constant et indépendant de l'obliquité de la vitesse sur la grille.

Si ce coefficient est égal à l'unité (grilles profilées), cette hypothèse rejoint la précédente. La question de son exactitude se posera done d'autant plus que ce coefficient sera plus différent de 1 , et le résultat risque alors de s'écarter plus de la réalité, au moins si on prend les valeurs correspondant à un écoulement normal.

En explicitant $h$, d'après ces expressions, on obtient successivement :

$$
\begin{gathered}
h=\left(\frac{d q}{d x}\right)^{2} \frac{1}{m^{2} 2 g \cos \alpha} \\
\frac{1}{h^{2}}=\left(\frac{d x}{d q}\right)^{4} 4 g^{2} m^{4} \cos ^{2} \alpha
\end{gathered}
$$

ce qui donne finalement l'équation différentielle :

$$
\begin{aligned}
\left(\frac{d q}{d x}\right)^{6}-2 g m^{2} \cos \alpha(x \sin \alpha & +\mathrm{H})\left(\frac{d q}{d x}\right)^{4} \\
& +4 g^{2} q^{2} m^{6} \cos ^{3} \alpha=0
\end{aligned}
$$

C'est une équation du premier ordre et du sixième degré, liant $q \dot{a} x$. Si on pouvait l'inté$\operatorname{grer}\left({ }^{*}\right)$, on aurait la longueur nécessaire en fonction de a et du débit en égalant l'intégrale à zéro (débit déversé nul).

\section{$2^{\circ}$ Considerations pratioutes}

De toutes facons, il est intéressant de trouver une méthode permettant de résoudre le pro-

\footnotetext{
* M. Kuntranan, professeur à luniversité de Grenoble, nous a indiqué le moyen de traiter cette question. Les calculs sont achuellement en cours.
}

blème. Nous avons envisagé un procédé par approximations sucessives qui converge d'ailleurs assez rapidement.

a) Etant donné le tirant d'eau ef la vilesse au point où les grilles commeneent (si te lit du torrent à l'amont immédiat des grilles est plat, on pourra admettre qu'on a, à cet endroit, la vitesse et la hauteur critiques), on trace d'abord une ligne d'eau fictive, en supposant que la grille ne débite pas, en appliquant le théorème des forces vives.

b) A partir de cette ligne d'eau fictive, on calcule, à l'aide des hypothèses précédentes, le débit qui passerait à travers la grille en supposant réalisée la charge précédente. La charge étant plus grande que celle réalisée en pratique, on trouvera un débit par excès.

c) Ce calcul fait, on connait, en fonction de l'abscisse $x$ suivant les grilles, le débit restant. Une nouvelle application du théorème des forces vives, avec les nouveaux débits, donne en chaque point les nouvelles hauteurs satisfaisant à la condition de conservation de l'énergie pour l'eau déversée.

Il ne reste plus qu'à recommencer le calcul $c$ ) avec les nouvelles hauteurs, qui donneront cette fois un débit prélevé trop faible.

On voit donc que les courbes successives obtenues par cette méthode seront situées successivement de part et d'autre de la courbe finalc.

Pratiquement d'ailleurs, l'application que nous avons faite de cette méthode à une prise auxiliaire de la chute d'Aussois nous a montré que le calcul convergeait très rapidement, surtout si on trace les lignes d'eau par tronçon de l'amont vers l'aval.

Ce calcul peut servir :

1) à déterminer la longueur minimum de grille nécessaire au captage d'un débit donné:

2) à đéterminer le débit qui s'introduira réellement dans la prise, lors d'une crue amenant la grille à ne pas absorber tous les apports.

\section{Conclusion}

Si le caractère aléatoire des hypothèses de base du calcul nous empêche de considérer le problìme comme traité de façon rigoureuse, du moins peut-on espérer tirer de ce calcul des renseignements pratiques utilisables dans de bonnes conditions : il appartiendra à l'expérience, qui garde toujours le dernier mot, de nous le confirmer.

\section{Bouvand,}

TNGÉNHUR A L'LUECTRICTE DE FRANCE REH Alpes II. 